\title{
Significance of the local sheet curvature in the prediction of sheet metal forming limits by necking instabilities and cracks
}

\author{
Pavel Hora ${ }^{1, a}$, Longchang Tong ${ }^{1}$, Maysam Gorji, Niko Manopulo ${ }^{1}$ and Bekim Berisha ${ }^{1}$ \\ ${ }^{1}$ ETH Zurich, Institute of virtual Manufacturing, Tannenstrasse 3, 8092, Zurich, Switzerland
}

\begin{abstract}
The industrial based prediction in sheet metal forming bases still on the Forming Limit Diagrams (FLD) as formally proposed by Keeler 1 . The FLD are commonly specified by the Nakajima tests and evaluated with the so called cross section method. Although widely used, the FLC concept has numerous serious limitations. In the paper the influences of bending on the FLC as well as the later crack limits will be discussed. Both criteria will be combined to an extended FLC concept (X-FLC). The new concept demonstrates that the Nakajima tests are not only appropriate for the evaluation of the necking instability but for the detection of the real crack strains too. For the evaluation of the crack strains a new local thinning method is proposed and tested for special 6xxx Al-alloys.
\end{abstract}

\section{Introduction}

Nowadays forming limits in sheet metal forming are mostly predicted based on the necking initiation. This limit will be usually evaluated with the Nakajima test. The FLC's are in this way only valid for linear strain path and for negligible curvature radii.

It is well known, that under conditions like hemming or in cases of deep drawing (DD) with small radii fracture limits arise, which needs different experimental as well as theoretical approaches.

Well known are the investigations of Hasek 3 who demonstrated the influence of different punch geometries on the position of the FLC, 0 .
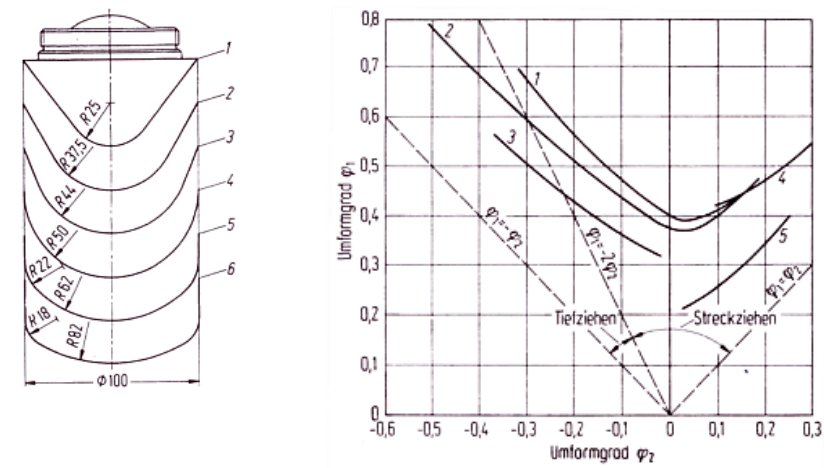

Figure 1 Influence of the curvature on the position of the FLC, left: used punch geometries. Hasek 3

Theoretical models like the M-K Model 2 do not consider the bending influence at all.

\section{Processes with bending impact on formability}

That the influence of the curvature is of industrial relevance can be demonstrated on different examples.

\subsection{Influence of bending effects on deep drawing behaviour}

0shows a deep drawing example with a die radius of $\mathrm{r}=3.0 \mathrm{~mm}$ for the material AA6016. The DD process was done with different square blanks and different blank holder forces.

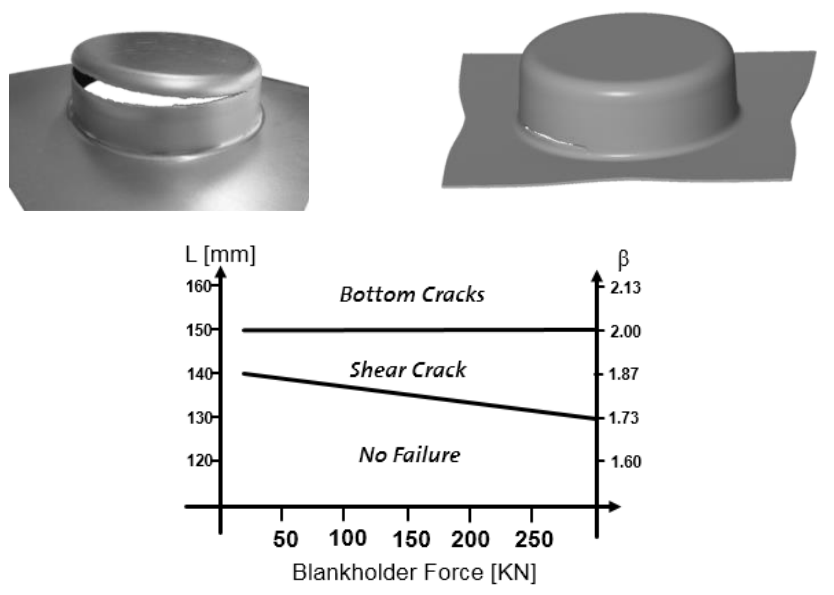

Figure 2 Formability diagram of AA6016 sheet sample, Gorji 17

\footnotetext{
${ }^{\mathrm{a}}$ Corresponding author: phora@ivp.mavt.ethz.ch
} 
Dependent on the selected parameter combination the localized necking occurs as classical bottom neck or as a upper radius crack. By increasing the die radius to $\mathrm{r}=5.0 \mathrm{~mm}$ the upper neck disappears.

Gorji 17 demonstrated that a FEM simulation based on shell elements and classical FLC is not able to predict this influence correctly.

\subsection{Prediction of DD behaviour of multilayer materials}

A more complex behaviour is given when multi-layer materials like the multilayer composite FUSION $^{\mathrm{TM}}$ [Novelis] is applied, 0 .
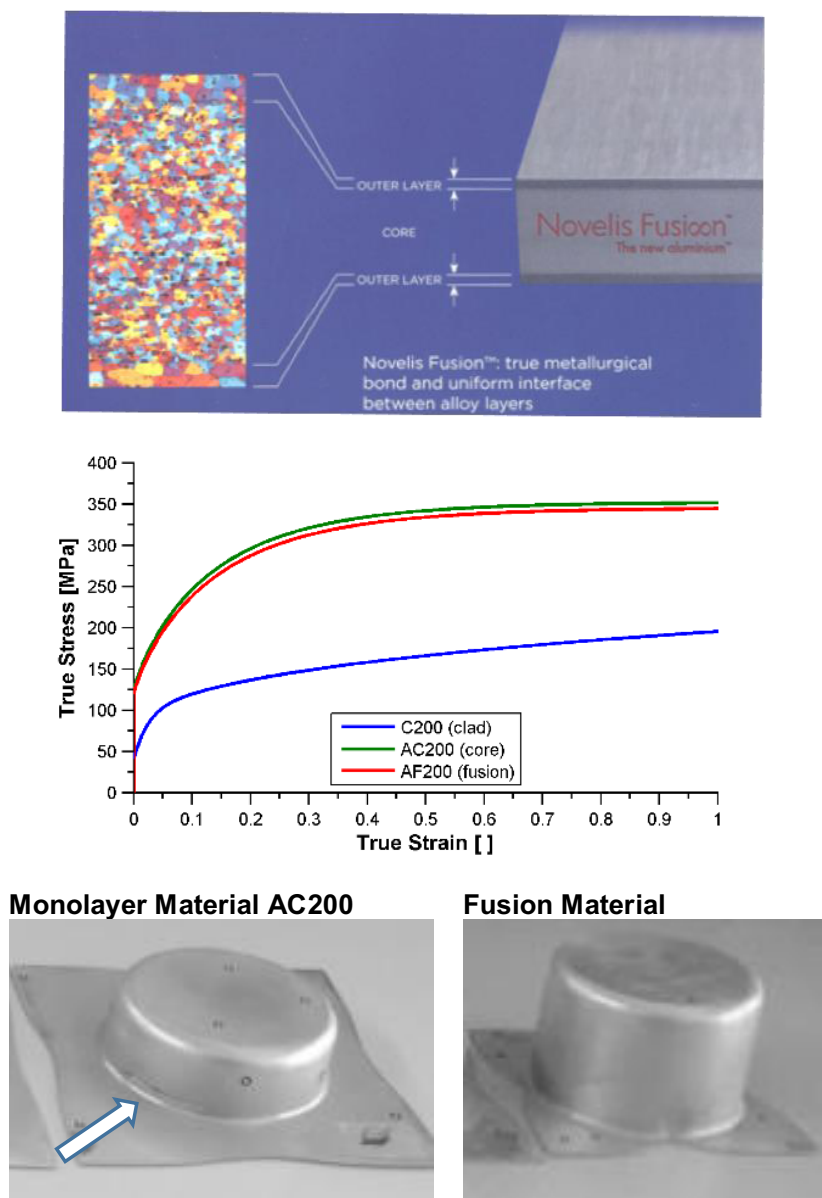

Figure 3 Multilayer FUSION material, yield curves. Influence on the forming behaviour. Gorji 17

The material is composed of a soft AA5005 alloy outside (clad) and a hard AA6016 alloy inside (core). 0bottom demonstrates again clearly how significantly the DD behaviour changes. Again a correct prediction cannot be done on the base of the classical FLC-method.

As reasons for the increase of the strain limits the change of the strain distribution in the sheet plane as well as strain gradients over the thickness can be seen as the influencing effects.

The thickness strain gradients depend on the ratio $\chi=t / r$. In the current DD examples with the die radius of $3.0 \mathrm{~mm}$ the critical ratio was about 0.3 .

\subsection{Prediction of crack limits for hemming operations}

From many former investigations it is well known, that in pure bending - corresponding to hemming operations the fracture strains lies even above the crack limits for flat sheets.
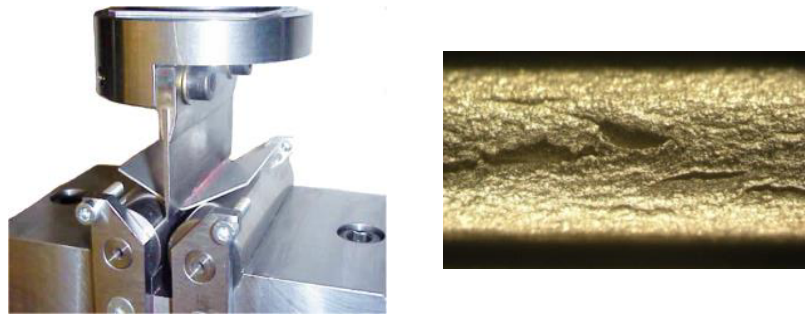

Figure 4 Evaluation of the fracture strain by bending tests. Occurrence of edge cracks (right). Gorji 17

0 demonstrates the bending results for the alloy AA6016 under different pre-strain conditions. It is worth to mention that the critical strain of the pure bending test surmount even the crack strains of the quasi flat Nakajima tests with $\chi=1 / 50=0.02$ by nearly $20 \%$.

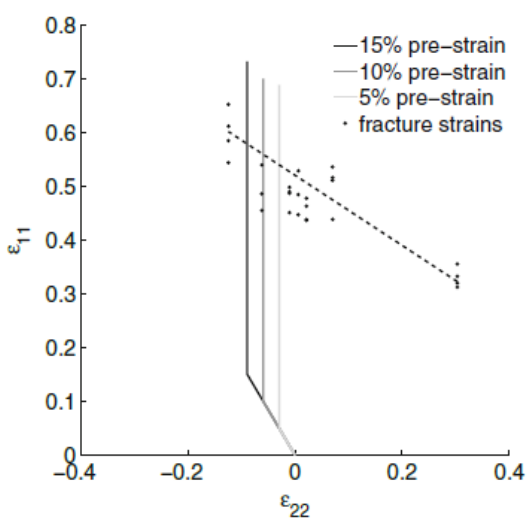

Figure 5 Fracture points determined by the Nakajima tests compared with the strains in bending at the critical state for AA6016. Gorji 16

\section{Prediction of FLC's under bending influences}

The already mentioned investigations by Hasek, 0predicts higher strains with increasing curvatures of the punches. This effect can be caused by stabilizing effects in the global strain distribution as well as by strain gradients in the thickness direction.

\subsection{MMFC criterion under consideration of strain rate and bending effects}

A basic description of the bending effect was formulated in the framework of the MMFC criterions by Hora et al.,5 to 12 and 18 .

The criterion can be expressed mathematically as 


$$
\frac{\partial \sigma_{11}}{\partial \varepsilon_{11}}+\frac{\partial \sigma_{11}}{\partial \beta} \cdot \frac{\partial \beta}{\partial \varepsilon_{11}}+\frac{\partial \sigma_{11}}{\partial \dot{\varepsilon}} \cdot \frac{\partial \dot{\varepsilon}}{\partial \varepsilon_{11}} \leq \sigma_{11}
$$

where $\beta=\Delta \varepsilon_{22} / \Delta \varepsilon_{11}$ denotes the strain increment ratio. Similarly, the stress ratio $\alpha$ is defined as $\alpha=\sigma_{22} / \sigma_{11}$. Despite of different descriptions of yield loci, the following relations can be generally established with functions:

$$
\begin{aligned}
& \sigma_{11}=f(\alpha) \bar{\sigma}, \text { and } \\
& \Delta \bar{\varepsilon}=g(\beta) \Delta \varepsilon_{11} .
\end{aligned}
$$

where $\bar{\sigma}$ and $\Delta \bar{\varepsilon}$ are the equivalent stress and the equivalent strain increment respectively.

With the definitions above, the description of the modified maximum force criterion takes the general form as

$$
H^{\prime} \leq \frac{f(\alpha)+\frac{f^{\prime}(\alpha) g(\beta) \beta}{\beta^{\prime}(\alpha) \bar{\varepsilon}}}{f(\alpha) g(\beta)} H
$$

where $H=f\left(\varepsilon_{v}\right)$ and $H^{\prime}=d \bar{\sigma} / d \bar{\varepsilon}$ are the functions for the description of hardening behaviours of the sheet materials.

\subsubsection{Influence of curvature}

If significant bending effects occurs the strain distribution is no more constant over the thickness. This effect was included into MMFC in following simplified way 4.

Setting the bottom layer as reference the strain distribution over the thickness can be expressed with a linear function:

$$
\Delta \varepsilon_{11}{ }^{\eta}=\Delta \varepsilon_{11}^{0}\left(1+\frac{\eta}{\rho_{0}}\right)
$$

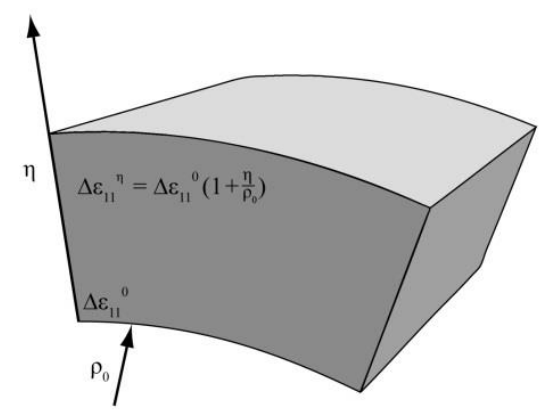

Figure 6 Strain distribution of a shell under bending conditiones

Substitute the relation into MMFC, the stress increment is expressed as:

$$
d \sigma_{11}=\frac{\partial \sigma_{11}{ }^{\eta}}{\partial \varepsilon_{11}{ }^{\eta}} d \varepsilon_{11}{ }^{\eta}+\frac{\partial \sigma_{11}}{\partial \beta} d \beta+\frac{\partial \sigma_{11}}{\partial \dot{\varepsilon}} d \dot{\varepsilon}
$$

Substitute (4) into (5) and make the approximation that

$$
\frac{\partial \sigma_{11}{ }^{\eta}}{\partial \varepsilon_{11}{ }^{\eta}} \approx \frac{\partial \sigma_{11}}{\partial \varepsilon_{11}}
$$

(5) can be transformed into:

$$
d \sigma_{11}=\frac{\partial \sigma_{11}}{\partial \varepsilon_{11}} d \varepsilon_{11}\left(1+\frac{\eta}{\rho_{0}}\right)+\frac{\partial \sigma_{11}}{\partial \beta} d \beta+\frac{\partial \sigma_{11}}{\partial \dot{\varepsilon}} d \dot{\varepsilon}
$$

Rewrite the original maximum force equation $d \sigma_{11} A+\sigma_{11} d A=0$ into the integral form

$$
\frac{\partial \sigma_{11}}{\partial \varepsilon_{11}} d \varepsilon_{11} \int_{0}^{t}\left(1+\frac{\eta}{\rho_{0}}\right) B d \eta+\left(\frac{\partial \sigma_{11}}{\partial \beta} d \beta+\frac{\partial \sigma_{11}}{\partial \dot{\varepsilon}} d \dot{\varepsilon}\right) A+\sigma_{11} d A=0
$$

where $A=B t$ is the current section area of the sheet. The final form of MMFC with the consideration of curvature and strain rate effects is obtained as:

$$
\frac{\partial \sigma_{11}}{\partial \varepsilon_{11}}\left(1+\frac{t}{2 \rho_{0}}\right)+\frac{\partial \sigma_{11}}{\partial \beta} \cdot \frac{\partial \beta}{\partial \varepsilon_{11}}+\frac{\partial \sigma_{11}}{\partial \dot{\varepsilon}} \cdot \frac{\partial \dot{\varepsilon}}{\partial \varepsilon_{11}} \leq \sigma_{11} .
$$

The influence of the curvature is shown later in 0 .

\subsubsection{Influence of strain rate}

It is well-known that steel materials in contrast to Alalloys have a positive strain rate dependency. The positive strain rate effects significantly slows down the necking and leads consequently to higher FLC values.

The description of strain rate effect in the framework of the analytical MMFC criterion - which bases on material point evaluations - requires a functional description of the strain rate increase in function of the equivalent strain, eq. (11).

A preliminary approach of the strain rate function can be defined by the $1 \mathrm{D}$ tensile conditions

$$
\begin{aligned}
& \varepsilon_{11} \leq n: \quad \dot{\varepsilon}=\dot{\varepsilon}_{\mathrm{hom}} \approx v / L \\
& \varepsilon_{11}=2 n: \quad \dot{\varepsilon}=\dot{\varepsilon}_{l o c} \approx v / 2 t
\end{aligned}
$$

A more general material specific description of the strain rate evolution by the terms of eq. (11) can be achieved by mapping the localization strain rate of the Nakajima tests. 0shows the history of the strain rate for different Nakajima specimens for the material DC05. 

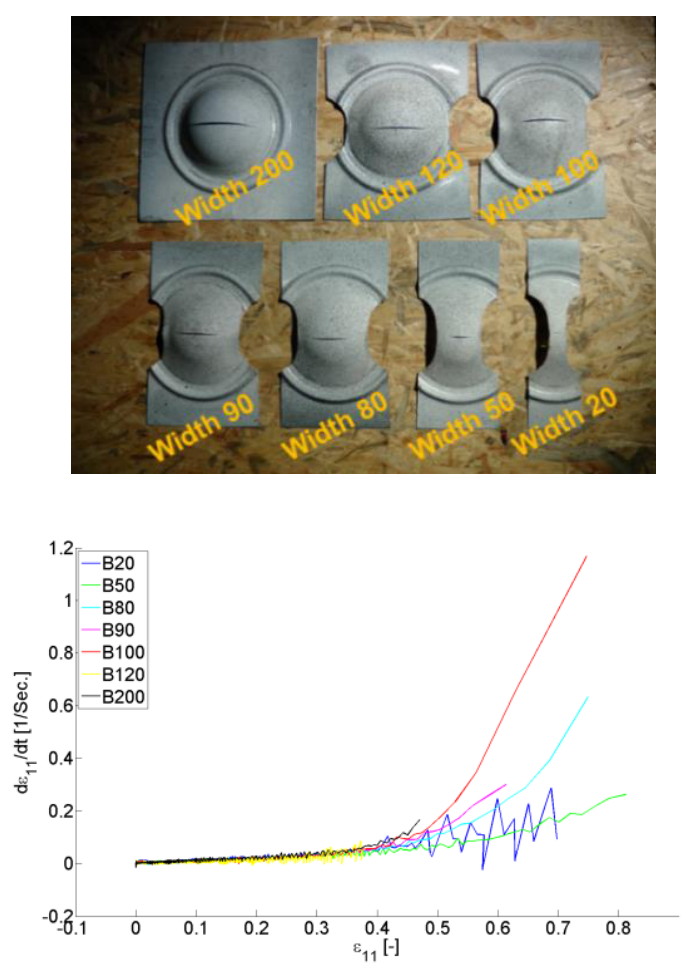

Figure 7 Increase of the strain rate for different shapes of Nakajima specimens. Material DC05

In a very approximative way the strain rate behaviour can mapped with the function

$$
\dot{\varepsilon}_{11}=\dot{\varepsilon}_{11}^{\text {hom }}+A(\beta) *\left[\left(\varepsilon_{11}-\varepsilon_{11}^{u n i}\right) / \varepsilon_{11}^{u n i}\right]^{p}
$$

Due to the fact, that the strain rate evolution varies very strongly for the different sizes of the Nakajima specimens $A(\beta)$ was fitted based on the measured strain rates of 0 and is given in 0 . Parameter $\mathrm{p}$ was assumed as $\mathrm{p}=2$.

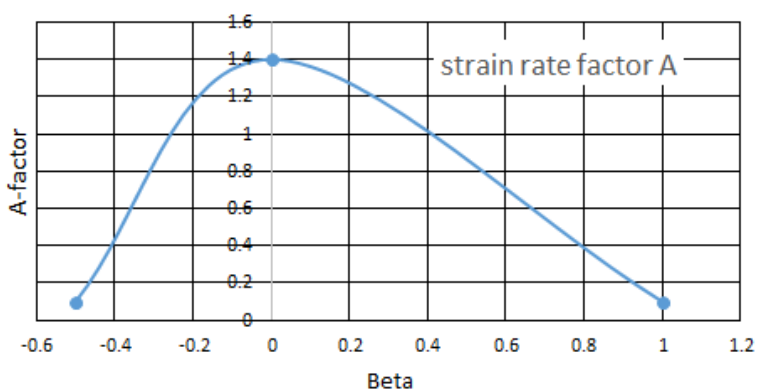

Figure 8 Distribution of the strain rate factor $A(\beta)$ in eq. (10) for a DC05 material.

In further investigations it shall be verified, if the same $A(\beta)$-Function can be used for specific materials groups.

\subsubsection{Validation for DC05}

The numerical evaluation of the MMFC, eq. (8), bases on an incremental calculation. The strain rate influence can then be evaluated directly based on the both dependencies $\dot{\varepsilon}_{e q}=g\left(\varepsilon_{e q}\right)$

$\sigma_{y}=f\left(\varepsilon_{e q}, \dot{\varepsilon}_{e q}\right)$

Material DC05 14:

$\sigma_{y}=\left[670.4 *(0.01+\varphi)^{0.174}-146\right](\dot{\varepsilon}+0.001)^{0.05}$

R-values:

\begin{tabular}{|l|l|l|l|l|}
\hline & $\mathrm{R}_{0}$ & $\mathrm{R}_{45}$ & $\mathrm{R}_{90}$ & $\mathrm{R}_{\mathrm{b}}$ \\
\hline $\mathrm{DC} 05$ & 2.1 & 1.5 & 2.5 & 0.85 \\
\hline
\end{tabular}

Yield stresses:

\begin{tabular}{|l|l|l|l|l|}
\hline & $\mathrm{Y}_{0}$ & $\mathrm{Y}_{45}$ & $\mathrm{Y}_{90}$ & $\mathrm{Y}_{\mathrm{b}}$ \\
\hline DC05 & 154.4 & 158.9 & 156 & 192 \\
\hline
\end{tabular}

The FLCs evaluated for the material DC05 are shown in 0 .

0top shows the experimental values of DCO5. Under the consideration of the strain rate influence as well as without it are visualized. 0bottom demonstrates the influence of the curvature as predicted with the MMFC approach. The different curves shows that an increase of FLC due to curvature becomes significant only for relatively large curvatures. For $\chi \leq 0.1$ the effect can be neglected
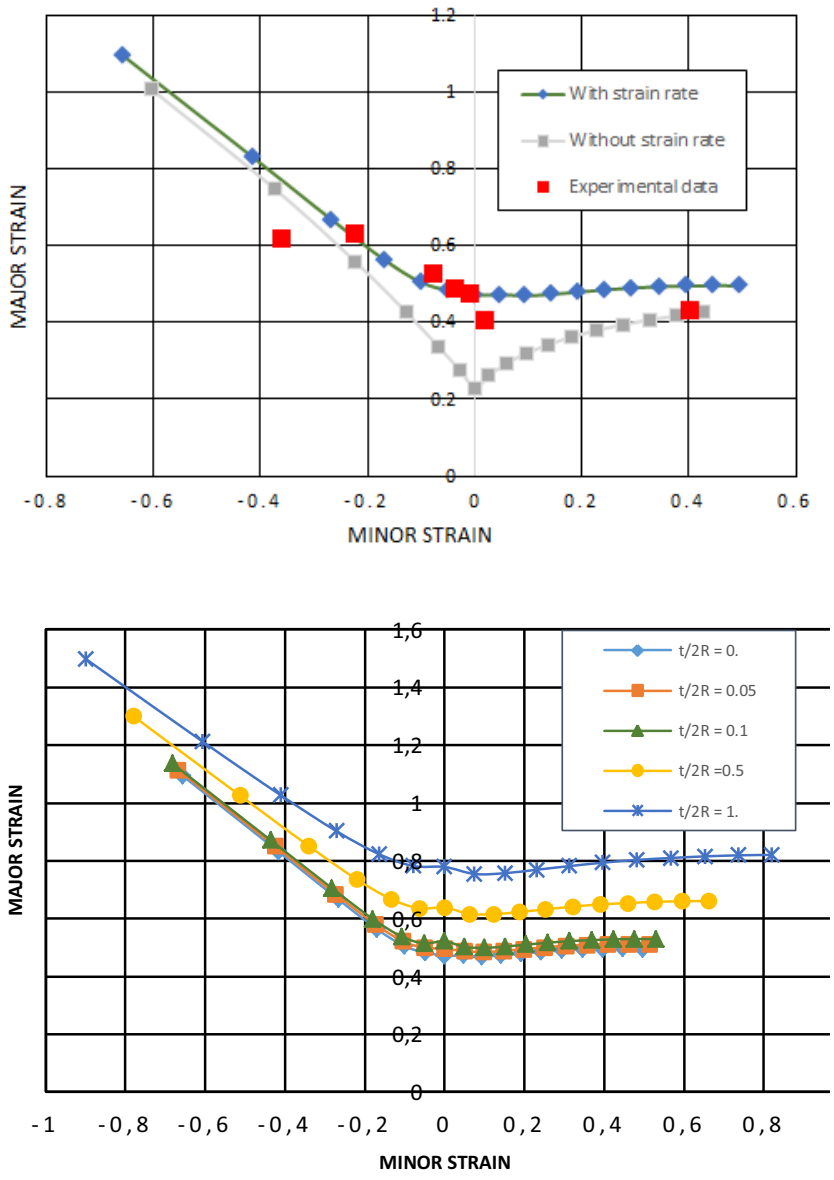

Figure 9 MMFC prediction of FLC's for a DC05 material. Top: FLC prediction with and without strain rate influence. Bottom: Influence of curvatures 


\section{Prediction of forming limits by cracks with and without bending effects}

\subsection{Experimental detection of crack limits based on Nakajima tests}

As demonstrated with the bending test and also related to other DD test with overlaid bending effects, not only the onset of necking instability but also the final crack have to be detected.

For the experimental detection of crack strains for sheet recent investigations by Wierzbicki, Bao, Bai and others 15 found a large interest. The authors proposed up to 15 different specimens to evaluate the so called triaxiality diagrams. As shown in 0.the proposed specimens have a very complex geometry which are difficult to be realized with sheet materials.
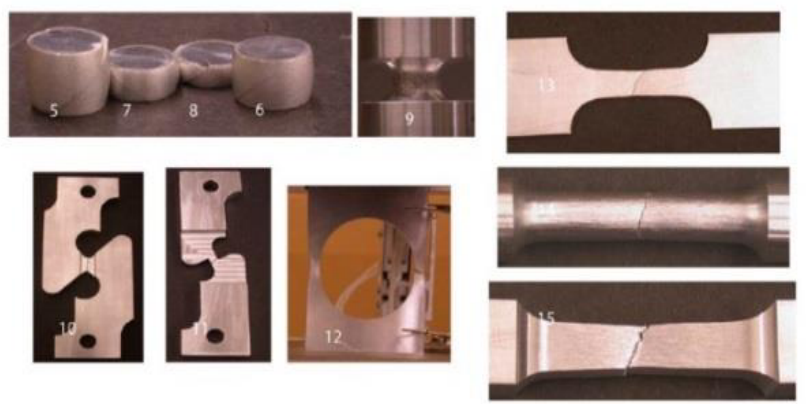

Figure 10 Proposed specimens for the evaluation of the triaxiality diagrams, 15

An alternative procedure was recently proposed by Gorji et al. 19. He uses a so called thinning method, where he evaluates with micrographs the crack thinning strain directly on broken Nakajima specimens, 0

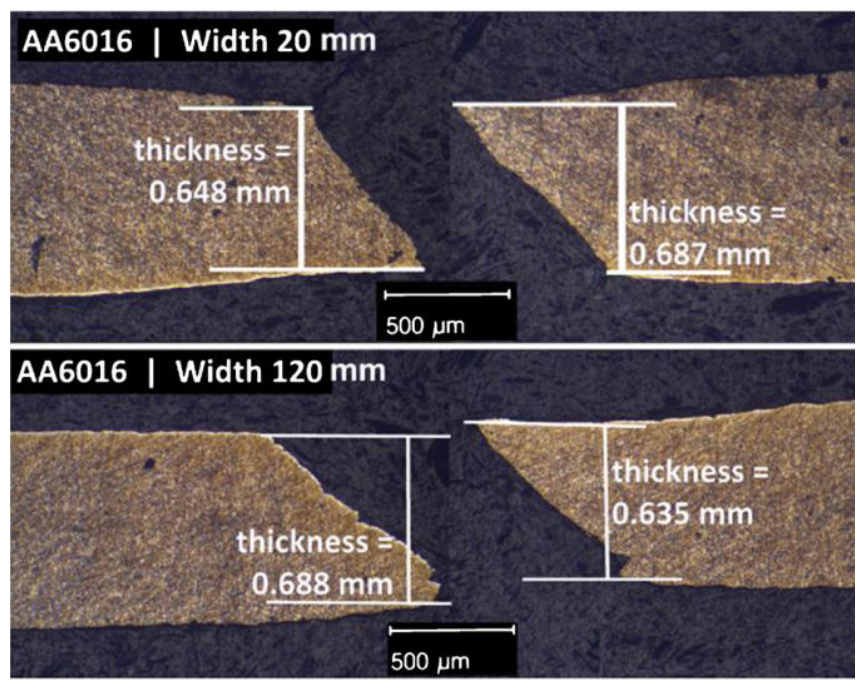

Figure 11 Evaluation of the fracture strain by the local detection of fracture thinning on the Nakajima specimens. Gorji 16,17

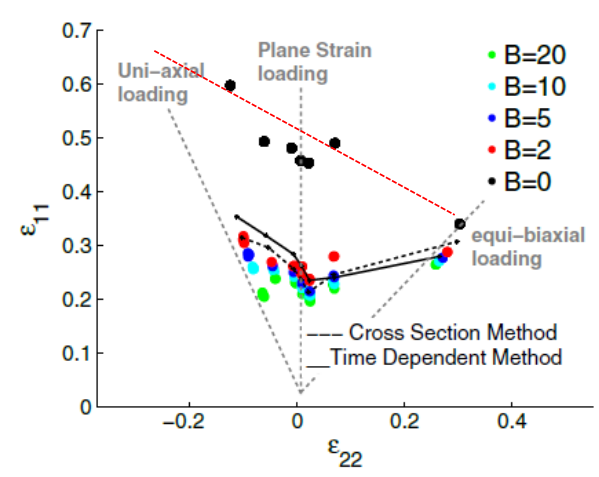

Figure 12 Evaluation of the fracture strain by the local detection of fracture thinning on the Nakajima specimens and comparison with the FLC. Gorji 16,17

\subsection{Experimental detection of crack limits based on Deep Drawing tests}

The Nakajima based test are restricted to the stress range

$$
0 \leq \alpha=\frac{\sigma_{2}}{\sigma_{1}} \leq 1.0
$$

In many deep drawing applications the largest strains occur on the left side of the FLC. For those reasons a special DD test with a quadratic blank and a relatively small die curvature of $\mathrm{r}=3.0 \mathrm{~mm}$ was applied to get an additional "point" specifically in the deep drawing (compression-tension combination) range. The experiment with the corresponding strain distribution at the crack time step shows 0

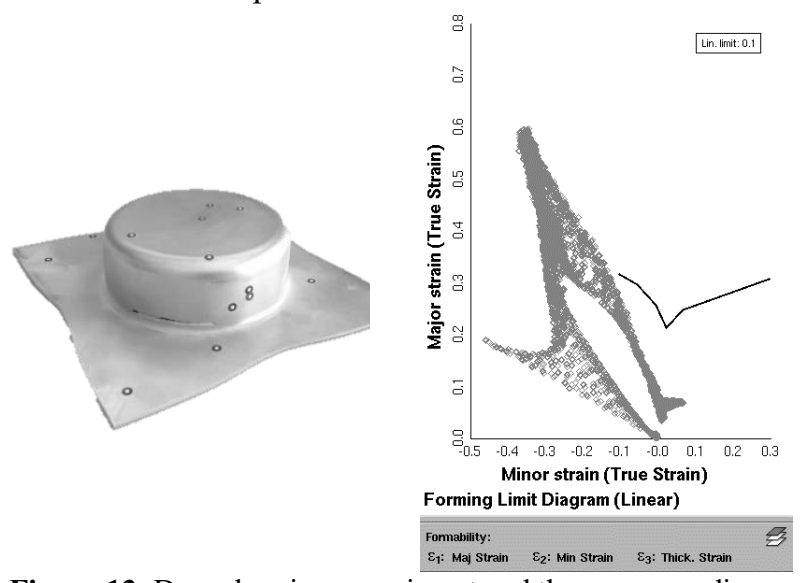

Figure 13 Deep drawing experiment and the corresponding strain distribution at the crack time step. Gorji 17

The combination of the Nakajima fracture strains with the additional DD fracture strains can be used as data base for the determination of a generalized fracture line.

\subsection{Comparison with different failure criteria}

The so experimentally predefined strain have been compared with the theoretical limits of different failure 
criteria. 0shows the fits of the theoretical fitted Failure curves with the experimental points. Remarkable is the fact, that all criterions fits the position of the measures points quite well, but that the criterions deviate significantly in the left "deep drawing" range. Especially this range influences strongly the virtual results for deep drawing operations.
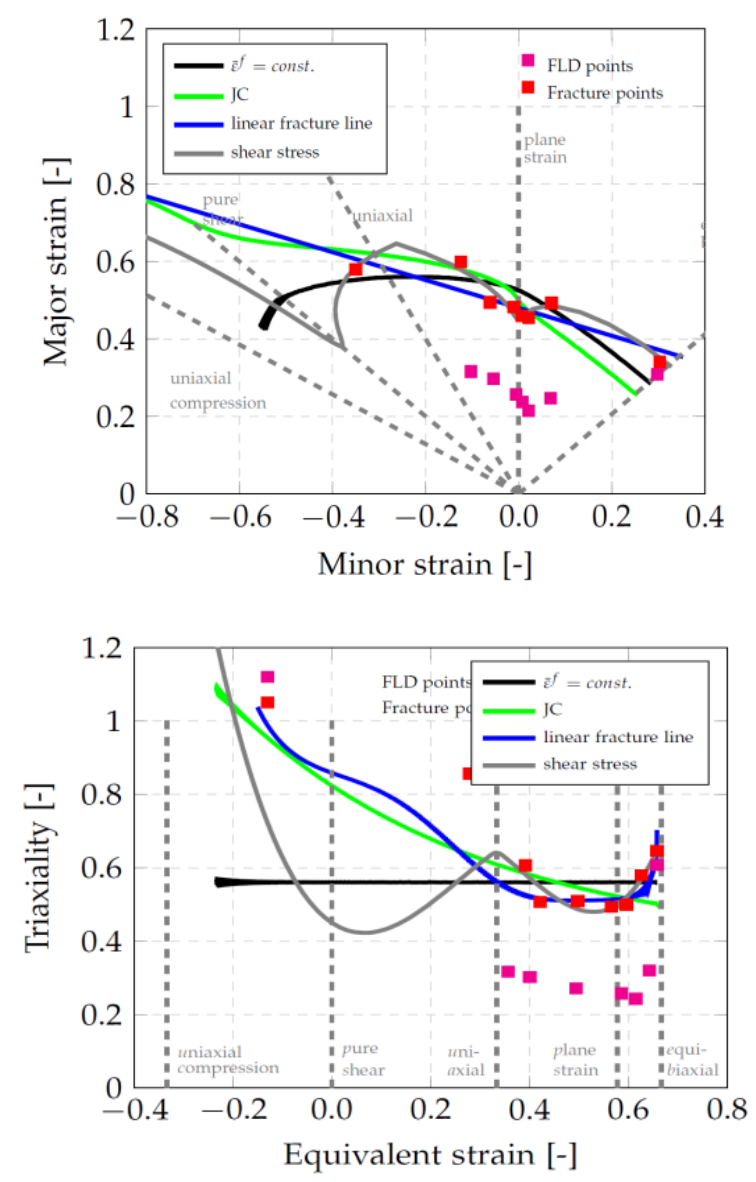

Figure 14 Comparison of different fracture criteria in principal strain space and in triaxiality-equivalent strain space, 17

Gorji et al. 17 demonstrated on comparisons with real parts that the Linear Fracture Line describes the behaviour in the most accurate way.

\section{Multilayer based consideration of the failure prediction}

\subsection{Layer based failure prediction with shell elements}

The classical failure predictions bases on a mono-layer FLC prediction. Typically the range above the FLC will be - from the real material perspective incorrectly considered as crack region.

If the fracture is initiated by a surface crack, as it is by small $\mathrm{t} / \mathrm{r}$ ratios, an extended X-FLC method has to be applied. The crack develops and initiates only when the outer layer reached the crack limit

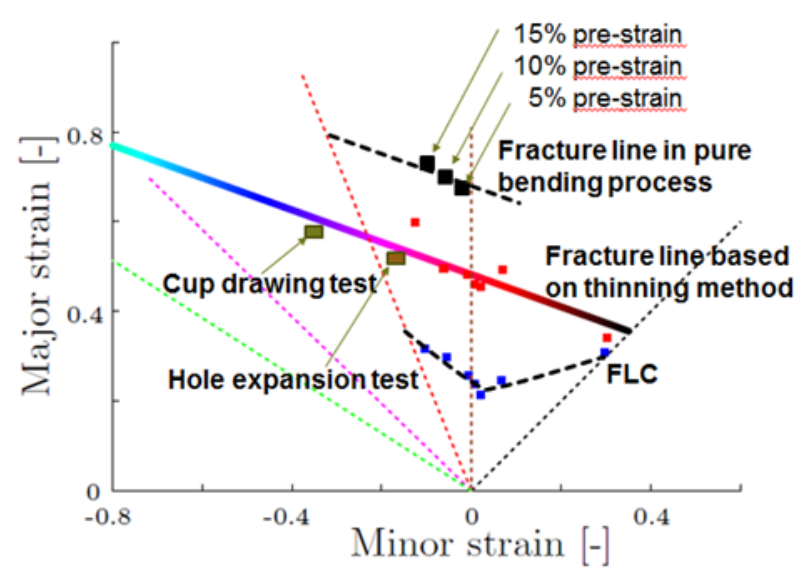

Figure 15 Extended X-FLC description considering the fracture ("crack") limits by the bending strains

\subsection{Prediction of crack limits for parts with small die radii and a monolayer structure}

For the validation of the above implementation a so called "triangle" test part was used.

Fig. 16 shows the crack position on the real part. The critical depth was at $\mathrm{H} \approx 43 \mathrm{~mm}$

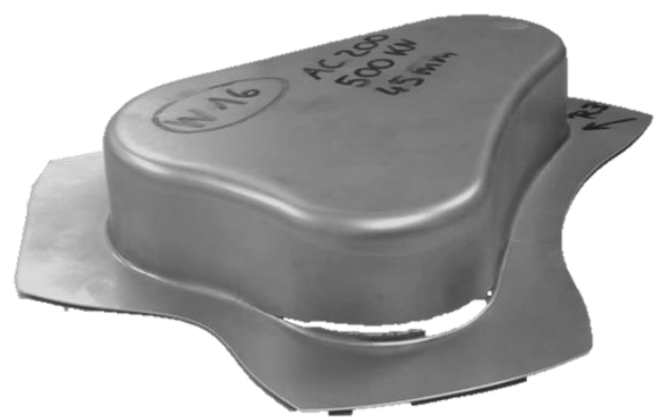

Figure 16 Triangle part. Monolayer material AA6016. Critical depth $\mathrm{H}=43 \mathrm{~mm}$

In the case of a simple "monolayer" FLC evaluation the predicted forming depth lies at $\mathrm{H}=29 \mathrm{~mm}$. It is obvious that not only the predicted part depth of $29 \mathrm{~mm}$ instead of $43 \mathrm{~mm}$ but also the position of the crack are not correctly predicted.

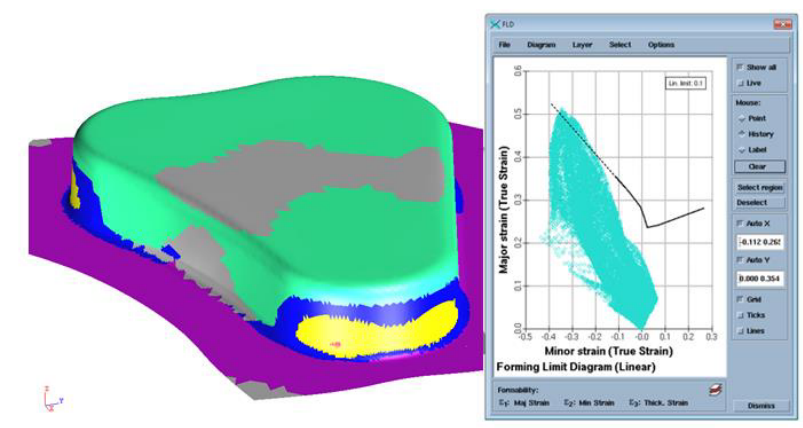

Figure 17 Triangle part. Monolayer material AA6016. Prediction of critical depth based on a monolayer FLC: $\mathrm{H}=29$ $\mathrm{mm}$

With the alternative method - applying the extended $\mathrm{X}$-FLC, 0 - the failure can be identified correctly, 0 

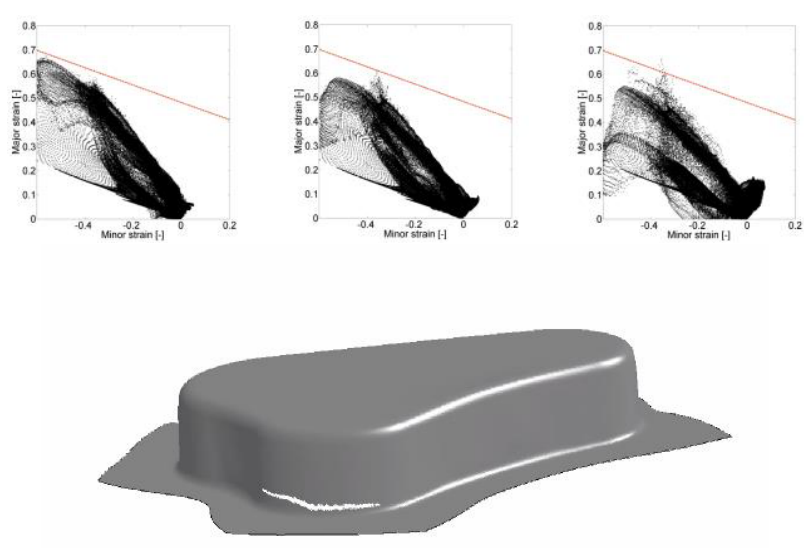

Figure 18 Material AA6016. FE Simulation of triangular experiment with Linear fracture criterion (strain distribution of lower, middle and upper-layer)

\subsection{Prediction of crack limits for multilayer FUSION materials}

If the monolayer material is replaced by the FUSION multilayer material as introduced in 0 , the failure can be completely avoided.

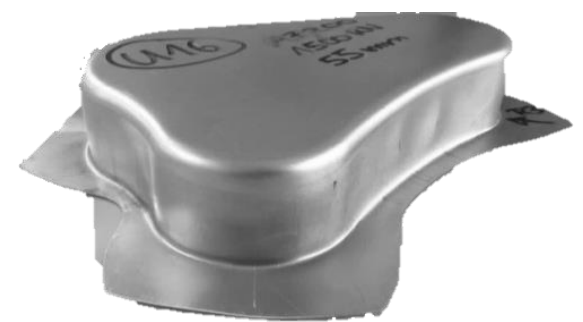

Figure 19 Material FUSION. Experiment with no fracture

For the simulation of the FUSION behaviour the XFLC diagrams have to be specified separately for the core and clad material. Interesting in this case is that the FLC's are the same for the soft (clad) and the hard (core) materials but the fracture lines reach strongly different levels, 0 .

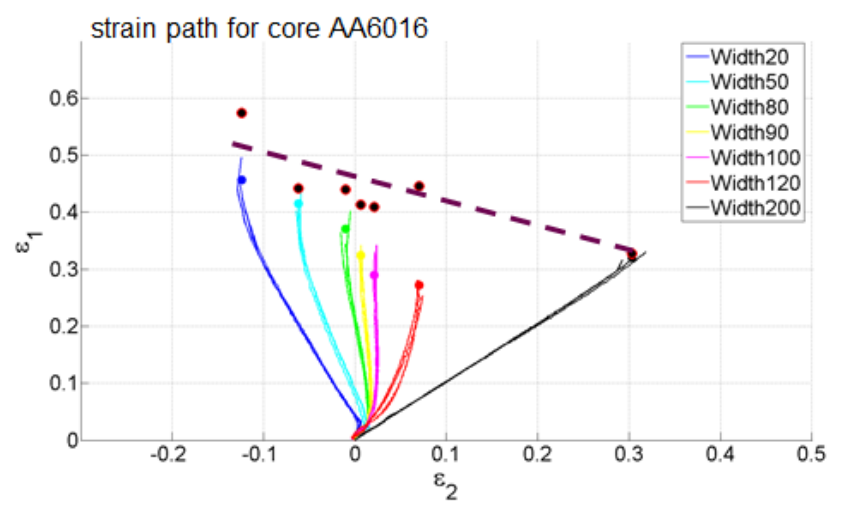

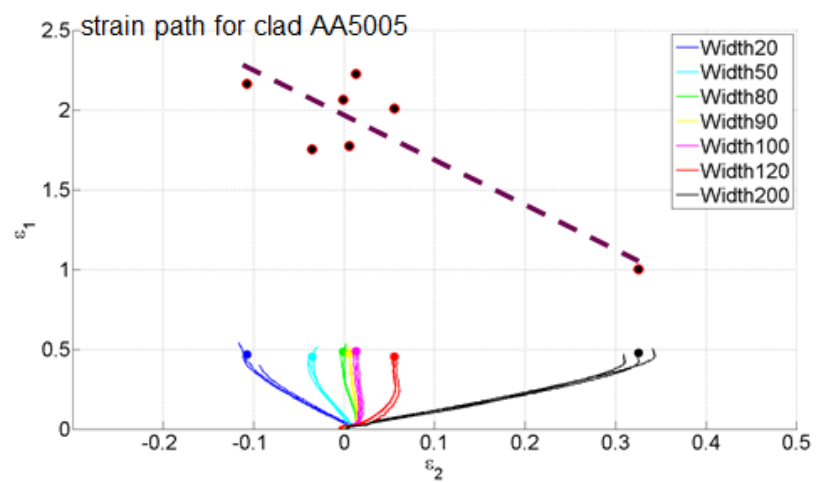

Figure 20 Comparing of strain history and fracture line of core and clad material.
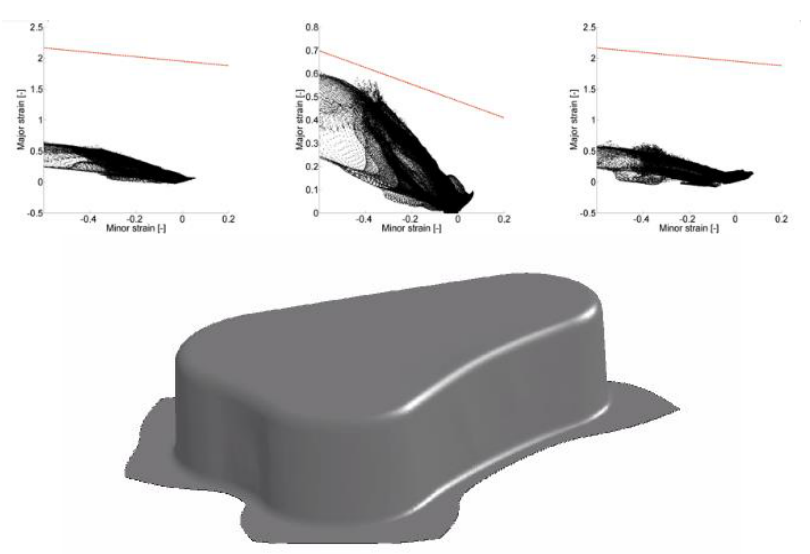

Figure 21 Forming behaviour of FUSION material: FE simulation of triangular experiment with linear fracture criterion specifically defined for the layers (strain distribution of lower, middle and upper-layer), Gorji 16.

\subsection{Prediction of crack limits for hemming operations}

The experimental detected crack limits based on the Nakajima test corresponds to the curvature of $\chi=t / r=1.0 / 50=0.02$. In this case the curvature can be practically neglected. In cases like the pure bending with $\chi=t / r=1.0 / 1.0=1$. the conditions are strongly different.

\subsubsection{Dependency of crack limits on curvature}

As demonstrated in 0experimentally evaluated strains lie above the fracture strain detected by the nearly flat Nakajima $(\chi=0.02)$ test.

Simulation of bending limits based on the previously determined X-FLC (Nakajima-cup limits) leads to a weak underestimate of the critical closing angle $\left(92^{\circ}\right.$ instead of $\left.74^{\circ}\right)$ 


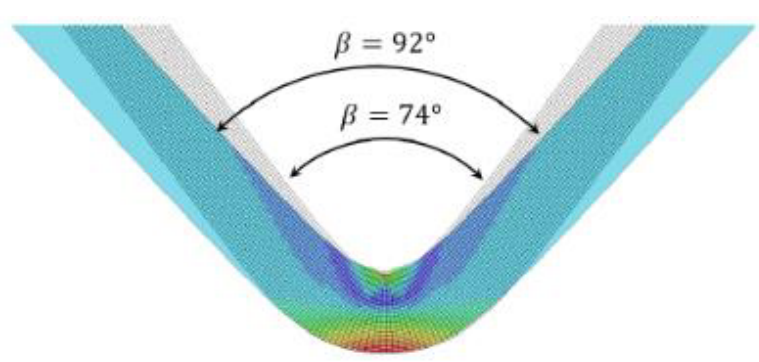

Figure 22 Evaluation of the fracture strain by the local detection of fracture thinning on the Nakajima specimens, Gorji 16.

For a better approach Hora and Gorji proposed a following correction. Correlation between membrane and bending fracture can be modelled by following relationship:

$$
\bar{\varepsilon}^{\text {Bending }}=\bar{\varepsilon}^{f^{\text {Membrane }}+c \chi}
$$

where $\chi=t / \rho$. Parameter c can be defined based on the linear graph in 0
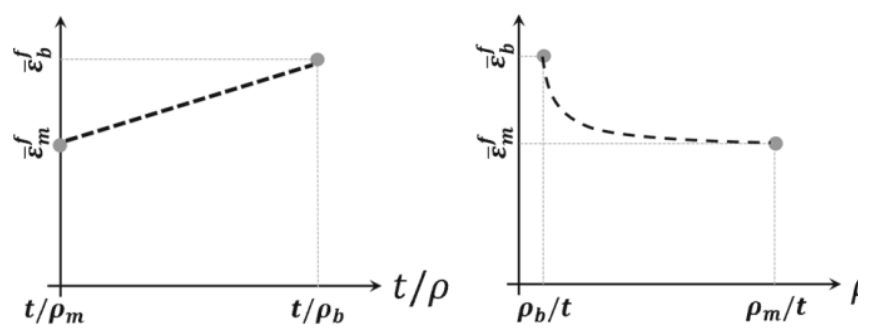

Figure 23 Correlation between membrane and bending fracture; $\rho_{m}$ and $\rho_{b}$ are punch radii in the Nakajima and 3-point bending experiment, respectively, Gorji 16 .

\section{Conclusions}

The contribution demonstrates on selected examples that the classical FLC prediction is not applicable if the parts have either small die radii or are composed by layers with different properties.

In this case beside the FLC a crack limit curve has to be specified too. For the detection of such critical strain the recently presented thinning method, evaluating the fracture strains on Nakajima specimens, have been used.

The multi-layer failure identification was implemented in to the explicit FEM code LS-Dyna.

\section{Acknowledgements}

The authors are very grateful to the CTI (The Swiss Innovation Promotion Agency) for the financial support of part of this work within the project 13082.1 PFIW-IW. Also thanks to J. Timm and E. Combaz (Novelis Switzerland SA) for providing the material and performing a part of the experiments. K. Wiegand (Daimler AG), M. Selig (AutoForm Development GmbH and E. Müller (GOM International AG) are also gratefully acknowledged for their contribution on the project results.

\section{References}

1. S. P. Keeler, Soc. of Automotive Engineering, Nr. 650535 , pp. 1-9, 1965.

2. Z. Marciniak and K. Kuczynski, Int. J. Mech. Sci., 9, pp. 609-620, 1967.

3. V.Hasek: Buch Lange Umfortmtech. Bd. 3, p.51-57

4. P. Hora et al: IFU-Krupp, Teilbericht II, 1993.

5. P. Hora et al:, IDDRG, 1994.

6. P. Hora, et al.:, NUMISHEET, Dearborn, MI, USA, pp. 252-256, 1996.

7. Tong, et al.:, NUMISHEET, Jeju, Korea, pp. 623627, 2002.

8. P. Hora et al.: Plasticity, Quebec, Canada, pp. 364366, 2003.

9. P.Hora, M. Merklein, L. Tong, J. Lechler:. Proc of IDDRG 2007

10. J. Krauer et al.: NumiForm Porto, Portugal, pp. 1263-1268, 2007.

11. P. Hora, T. Longchang:. Numisheet 2008

12. Numisheet Benchmark 1: Numisheet, Interlaken, Switzerland, 2008.

13. S.P. Keeler: pp. 4. $2.12,1989$.

14. P.Peters: Diss. ETH 22707 (2015).

15. Y.Bai, T.Wierzbicki:. Int J Fract (2010) 161:1-20

16. M.Gorji, B. Berisha, P.Hora, F. Barlat: Int.J. of Mater. Form., DOI 10.1007/s12289-015-1242-y, 2015.

17. M.Gorji:. Diss ETH 23066, 2015

18. N. Manopulo, P. Hora, P. Peters, M. Gorji, F.Barlat: Int. J. Plasticity,75, 189-203, 2015

19. M. Gorji, B. Berisha, N. Manopulo, P. Hora. J Materials Processing Technology, 232: 19-33, 2016 